1 Universidade de São Paulo (USP), Escola de Enfermagem (EE), Departamento de Enfermagem em Saúde Coletiva (ENS) - São Paulo (SP), Brasil.

2 Universidade Federal de Pelotas (UFPel), Faculdade de Medicina, Curso de Terapia Ocupacional

- Pelotas (RS), Brasil.

\section{Práticas de Atenção Primária à Saúde na área de drogas: revisão integrativa}

\author{
Primary Health Care practices in the drugs area: integrative review
}

\author{
Luíza Carraschi de Oliveira' ${ }^{\mathbf{1}}$ Luciana Cordeiro ${ }^{\mathbf{2}}$, Cassia Baldini Soares $\mathbf{1}$, Célia Maria Sivalli \\ Campos ${ }^{\mathbf{1}}$
}

DOI: $10.1590 / 0103-1104202112920$

\begin{abstract}
RESUMO O objetivo deste estudo foi o de identificar e analisar as práticas voltadas ao consumo prejudicial de drogas na Atenção Primária à Saúde. Trata-se de Revisão integrativa que buscou estudos nas fontes Medline e Lilacs utilizando os termos ‘Atenção Primária à Saúde’ e ‘Redução do Dano'. Como resultado, incluiram-se 52 estudos, analisados de acordo com os arcabouços teóricos que orientam as práticas em saúde. Tais estudos foram sintetizados em três categorias empíricas: comportamento de risco, que incluiu intervenção breve, programas para prevenir e diminuir o uso de drogas, entre outros; fatores determinantes, que incluiu visitas domiciliares, práticas grupais e organizacionais; e necessidades em saúde, que incluiu práticas educativas emancipatórias. Conclui-se que, majoritariamente, os estudos abordam o uso de drogas pela categoria risco, com proposição de práticas para adaptação social. As intervenções relativas aos determinantes promovem a saúde, propondo melhorias em ambientes de vida e trabalho. Práticas críticas às relações sociais estabelecidas pelo complexo das drogas são minoritárias e envolvem complexidade operacional.
\end{abstract}

PALAVRAS-CHAVE Revisão. Atenção Primária à Saúde. Redução do dano.

\begin{abstract}
The objective was to identify and analyze practices in Primary Health Care to harmful drug consumption. This integrative review searched for primary studies in Medline and Lilacs databases, based on the terms 'Primary Health Care' and 'Harm Reduction'. We included 52 studies analyzed according to the theoretical frameworks that guide health practices. Results were summarized in three empirical categories: risk behavior, which included briefintervention, programs to prevent and / or reduce drug use, among others; determining factors, including home visits and group and organizational practices; health needs, which included emancipatory educational practices. Most studies address drug use by the risk category producing practices aimed at social adaptation. Interventions related to determinants seek to promote health by proposing improvements in living and working environments. Practices that criticize social relations established by the drugs circuitous are more complex and sparse.
\end{abstract}

KEYWORDS Review. Primary Health Care. Harm reduction. 


\section{Introdução}

O campo das políticas relacionadas à temática das drogas é complexo, tanto na dimensão teórica, que aborda a matéria a partir de diversas concepções, como na dimensão das práticas. Esse campo é alvo de constantes tensões e disputas no cenário internacional e no Brasil em particular¹.

Análise das políticas públicas sobre drogas a partir de documentos oficiais reitera o embate histórico entre os paradigmas proibicionista e antiproibicionista, na área da justiça ou segurança, e entre os modelos asilar - representados pelas comunidades terapêuticas e centros de internamento de longa permanência - e psicossocial - baseado na rede territorial -, na área da saúde. A Redução de Danos (RD), que agrega teorias e práticas, é considerada possível facilitadora de diálogo entre os setores envolvidos na problemática do uso prejudicial de drogas, quais sejam, saúde, justiça e segurança pública e assistência social1.

A RD é abordada a partir de distintos referenciais teóricos ${ }^{\mathbf{2}, \mathbf{3}}$, mesmo quando não estejam explícitos, no conjunto diversificado de práticas de RD orientadas pelas políticas de Estado. Esse conjunto diversificado alberga, num extremo, ações pragmáticas, restritas à distribuição de insumos para minimizar riscos individuais e, no outro, ações políticas complexas, que demandam a proteção do Estado e denúncias das desigualdades que estão na base do envolvimento de grupos sociais que vivem à margem da sociedade ${ }^{2}$.

No Brasil, pode-se reconhecer a incorporação da $\mathrm{RD}$ de forma mais ampla em documentos ministeriais de $2003^{4}$ e $2004^{5}$, embora historicamente note-se sua presença no cenário das políticas nacionais há mais tempo, com iniciativas locais que influenciaram as políticas de Estado de forma marcante ${ }^{6}$. Pautado pelo respeito aos direitos sociais e à autonomia do cidadão que usa drogas, o Ministério da Saúde brasileiro, em etapa progressista da política estatal, incorporou a RD na implantação de respostas mais adequadas ao enfrentamento do fenômeno do consumo de drogas, desafiando a perspectiva dominante, reconhecidamente servil à Guerra às Drogas (GD), de origem estadunidense ${ }^{7}$.

Revisão da literatura ${ }^{3}$ constatou na produção sobre $\mathrm{RD}$ a persistência de práticas de caráter pragmático, embasadas em referenciais teóricos pós-modernos, com a presença de ecletismo teórico ou não explicitação de referencial teórico. Com tamanha diversidade, mesmo podendo ser classificadas como progressistas, já que negam a repressão imposta pela GD, as práticas de $\mathrm{RD}$ não têm conseguido avançar suficientemente para alvejar os problemas que estão na base da mercantilização e fetichização de drogas.

No Brasil, são marcantes três tendências em disputa sobre a $\mathrm{RD}$, quando se toma em consideração o discurso de formadores de opinião na área. A concepção mais recorrente é a de que o consumo de drogas é uma doença e, portanto, os consumidores devem receber tratamento psiquiátrico para reabilitação e reinserção social, perspectiva essa amparada pelo arcabouço teórico e de práticas do campo da saúde pública. Outra tendência é a liberal, em que o consumidor de drogas é compreendido como portador de direitos individuais, sendo que as estratégias de $\mathrm{RD}$ têm como finalidade garantir cidadania e cuidados humanizados em saúde. A terceira é a tendência crítica, que interpreta o fenômeno produção-distribuição-consumo de drogas na sua subordinação ao modo de produção capitalista, e propõe a $\mathrm{RD}$ emancipatória como abordagem paradigmática, orientada pela perspectiva do campo da saúde coletiva ${ }^{8}$.

Resultados de metassíntese de relatos de experiências nacionais e internacionais sobre avanços e entraves de implantação revelaram que a RD é adotada nos serviços de saúde como estratégia que apoia ações de prevenção e tratamento, geralmente voltadas à melhoria das condições de vida, de saúde e de sobrevivência de dependentes de drogas, mantendo-os na rede de atenção à saúde e de assistência social com vistas a garantir direitos humanos e de 
inclusão social9, o que evidencia seu caráter progressista. Essa revisão 9 não explorou a especificidade das práticas de RD no âmbito da Atenção Primária à Saúde (APS) e reitera resultados anteriores que atestam a diversidade de fundamentação teórica da RD ou até mesmo sua ausência.

A partir dessas considerações, este estudo tem por objetivo identificar e analisar as práticas de $\mathrm{RD}$ voltadas ao consumo prejudicial de drogas no âmbito da APS e, mais especificamente, identificar as concepções que as orientam, para captar a diversidade de orientações que estão na base dessas práticas.

\section{Material e métodos}

Trata-se de Revisão Integrativa (RI), um tipo de síntese da literatura científica que permite ir além da agregação de resultados de estudos levantados de acordo com semelhança metodológica. A RI promove síntese integrada dos achados, independentemente de seu desenho metodológico, baseada em estrutura conceitual coerente para amparar a pergunta e o fenômeno de interesse, auxiliando no desenvolvimento de práticas inovadoras. Permite, ainda, que conceitos que amparam as práticas sejam analisados ${ }^{10}$.

A RI aqui adotada se fundamentou no materialismo histórico-dialético ${ }^{11}$.

A RI foi realizada em cinco etapas ${ }^{12}: 1$ ) Formulação da pergunta e objetivos da revisão; 2) Descrição da metodologia da revisão contendo o estabelecimento de critério de inclusão e exclusão; a definição de estratégia e equação de busca em bases de dados; e o detalhamento do processo de busca e seleção dos estudos; 3 ) Análise e interpretação dos dados provenientes dos estudos primários, onde se definem as informações a serem extraídas dos estudos primários e se descreve o processo de análise e síntese; 4) Apresentação dos resultados descrevendo as características dos estudos incluídos e apresentando em quadro com dados bibliométricos dos estudos; 5) Interpretação dos resultados, a partir do referencial teórico-metodológico adotado.

A busca foi realizada no primeiro semestre de 2018, e uma atualização foi realizada em fevereiro de 2020. Utilizaram-se as bases de dados Medline e Lilacs, acessadas por meio do portal BVS Saúde Pública, com a seguinte equação de busca: ("redução do dano" OR "harm reduction" OR "reducción del daño") OR (prevenção OR prevention OR prevención) OR (educação OR education OR educación)) AND (("drogas Ilícitas" OR "street drugs" OR "drogas ilícitas") OR ("bebidas alcoólicas" OR "alcoholic beverages" OR "bebidas alcohólicas")) AND (("saúde coletiva" OR "saúde pública" OR "public health" OR "salud pública") OR ("atenção básica à saúde" OR "atenção primária à saúde" OR "primary health care" OR "atención primaria de salud") OR ("community health services" OR "assistência à saúde comunitária") OR ("health maintenance organizations") OR ("medicina de família e comunidade" OR "family practice")) AND NOT (disease OR (hiv OR "acquired immunodeficiency syndrome” OR aids)).

Os critérios de inclusão elegeram estudos primários ou de revisão que descrevessem, analisassem, propusessem intervenções voltadas a usuários de drogas próprias para o contexto da APS. Não houve restrição temporal. Incluíram-se trabalhos publicados em português, inglês ou espanhol. Não foram incluídos artigos sobre: práticas de controle de doenças transmissíveis, como HIV, Hepatite C, entre outras; ações relacionadas à atenção a problemas psiquiátricos envolvendo consumo de psicoativos; práticas clínicas com o objetivo precípuo de tratamento; intervenções específicas para reposição de insumos; intervenções voltadas para populações específicas, como pessoas em situação de cárcere e gestantes; e ainda estudos de custo-benefício ou custo-eficácia.

Após a busca nas bases de dados, procedeu-se à leitura de título e resumo, conforme critérios de inclusão e exclusão e, em seguida, à leitura na íntegra dos estudos selecionados. 
Os estudos incluídos foram apresentados segundo o ano de publicação, país de origem, delineamento e objetivo. Para captar a diversidade de orientações das práticas na APS, procurou-se identificar as concepções que as orientam, por meio da caracterização do objeto das práticas na APS, compreendendo-se como objeto o que se deseja transformar por meio do trabalho em saúde 2 .

Estão registradas na figura 1 as etapas de identificação, seleção, elegibilidade e inclusão das publicações. Fluxograma adaptado ${ }^{13}$.

Figura 1. Fluxograma dos artigos incluídos na revisão integrativa
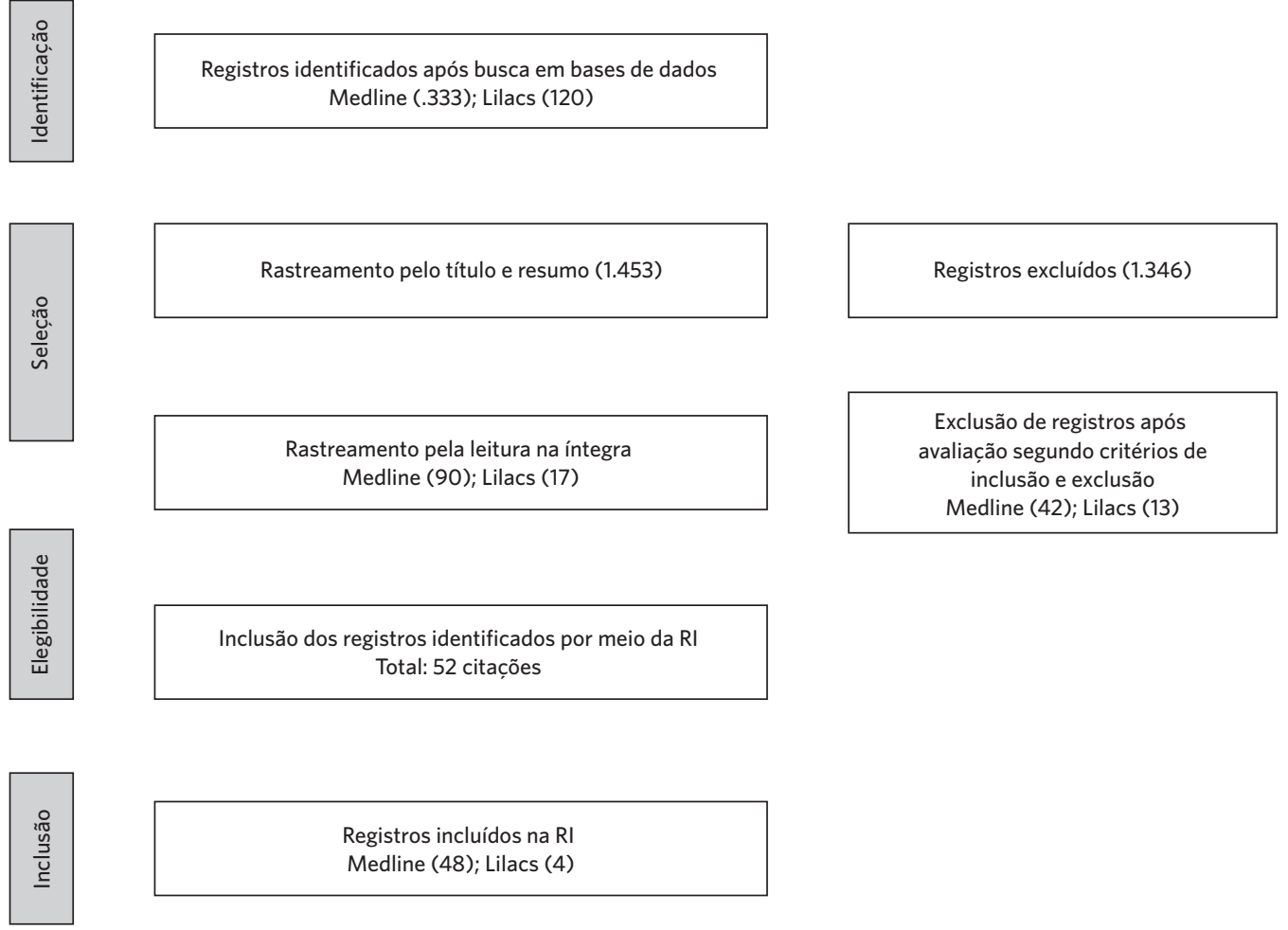

Registros incluídos na RI Medline (48); Lilacs (4)

Fonte: Moher D et al. ${ }^{\mathbf{1 3}}$

\section{Resultados}

Selecionaram-se 52 artigos identificados de um total de 1.453, sendo 48 da fonte Medline e 4 da Lilacs. Os dados bibliométricos estão sumariados no quadro 1. Quanto aos países de origem dos estudos, 23 provieram dos EUA (44,2\%), sete do Brasil (13,4\%), cinco da Austrália (9,6\%), quatro da Espanha (7,6\%), quatro do Reino Unido (7,6\%), três do Canadá (5,7\%), um da Dinamarca (1,9\%), um de Portugal (1,9\%), um da Finlândia (1,9\%), um da Suíça (1,9\%), um da Holanda (1,9\%) e um da Suécia (1,9\%). Todos foram publicados após 2003. Dos 52 artigos identificados 15 fizeram revisões da literatura (32,6\%). A base de dados Lilacs identificou quatro dos sete estudos brasileiros que compuseram o corpus desta revisão, sendo provenientes da área da enfermagem, principalmente, e da psicologia. 
Quadro 1. Caracterização dos estudos em relação aos autores, ano de publicação, título e objetivo do estudo, conforme a base de dados

\begin{tabular}{|c|c|c|}
\hline AUTOR & TÍTULO & OBJETIVO \\
\hline $\begin{array}{l}\text { Amaral, Ronzani, Sou- } \\
\text { za-Formigoni, } 2010\end{array}$ & $\begin{array}{l}\text { Process evaluation of the implementa- } \\
\text { tion of a screening and brief intervention } \\
\text { program for alcohol risk in primary heal- } \\
\text { th care: An experience in Brazil }\end{array}$ & $\begin{array}{l}\text { Avaliar o processo de implementação de um pro- } \\
\text { grama de SBI (Screening and Brief Intervention) } \\
\text { para o uso de risco de álcool e avaliar os fatores } \\
\text { que facilitaram ou impediram tal implementação. }\end{array}$ \\
\hline Anderson, 1993 & $\begin{array}{l}\text { Effectiveness of general practice inter- } \\
\text { ventions for patients with harmful alco- } \\
\text { hol consumption }\end{array}$ & $\begin{array}{l}\text { Avaliar a eficácia das intervenções realizadas } \\
\text { por médicos de clínica geral para indivíduos com } \\
\text { consumo prejudicial de álcool. }\end{array}$ \\
\hline Ballesteros et al., 2004 & $\begin{array}{l}\text { Efficacy of brief interventions for hazar- } \\
\text { dous drinkers in primary care: systematic } \\
\text { review and meta-analyses }\end{array}$ & $\begin{array}{l}\text { Avaliar a eficácia das IBs quando aplicadas em } \\
\text { ambientes de APS. }\end{array}$ \\
\hline Beich et al., 2007 & $\begin{array}{l}\text { Screening and brief intervention tar- } \\
\text { geting risky drinkers in Danish general } \\
\text { practice--a pragmatic controlled trial }\end{array}$ & $\begin{array}{l}\text { Avaliar a eficácia das recomendações da OMS } \\
\text { para triagem e intervenção breve (SBI) na clínica } \\
\text { geral. }\end{array}$ \\
\hline Bertholet, 2005 & $\begin{array}{l}\text { Reduction of alcohol consumption by } \\
\text { brief alcohol intervention in primary care: } \\
\text { systematic review and meta-analysis }\end{array}$ & $\begin{array}{l}\text { Avaliar a eficácia de intervenções breves com } \\
\text { álcool destinadas a reduzir o uso prolongado de } \\
\text { álcool e os danos relacionados em indivíduos que } \\
\text { frequentam unidades de APS. }\end{array}$ \\
\hline Bryant et al., 2007 & $\begin{array}{l}\text { Community-based prevention marketing: } \\
\text { organizing a community for health beha- } \\
\text { vior intervention }\end{array}$ & $\begin{array}{l}\text { Descrever a aplicação e o aprimoramento do } \\
\text { marketing de prevenção baseado na comunidade } \\
(\text { CBPM). }\end{array}$ \\
\hline Chun, Linakis, 2012 & Interventions for adolescent alcohol use & $\begin{array}{l}\text { Rever a literatura atual sobre prevenção de uso } \\
\text { de álcool em adolescentes e sobre estratégias de } \\
\text { intervenção. }\end{array}$ \\
\hline Córdoba et al., 1998 & $\begin{array}{l}\text { Effectiveness of brief intervention on } \\
\text { non-dependent alcohol drinkers (EBIAL): } \\
\text { a Spanish multi-centre study }\end{array}$ & $\begin{array}{l}\text { Comparar a eficácia de uma intervenção breve, } \\
\text { comparada a aconselhamento, para prevenção } \\
\text { do uso prejudicial de álcool. }\end{array}$ \\
\hline Curry et al., 2003 & $\begin{array}{l}\text { A randomized trial of a brief primary- } \\
\text {-care-based intervention for reducing } \\
\text { at-risk drinking practices }\end{array}$ & $\begin{array}{l}\text { Avaliar a intervenção para reduzir o consumo de } \\
\text { risco relacionado ao álcool em uma amostra de } \\
307 \text { indivíduos. }\end{array}$ \\
\hline $\begin{array}{l}\text { Elzerbi, Donoghue, } \\
\text { Drummond, } 2015\end{array}$ & $\begin{array}{l}\text { A comparison of the efficacy of brief } \\
\text { interventions to reduce hazardous and } \\
\text { harmful alcohol consumption between } \\
\text { European and non-European countries: a } \\
\text { systematic review and meta-analysis of } \\
\text { randomized controlled trials }\end{array}$ & $\begin{array}{l}\text { Determinar a eficácia geral da IB, após seis e } \\
\text { doze meses de acompanhamento em estudos } \\
\text { em (APS) e em departamento de emergência } \\
\text { (DE); examinar se a variação no resultado do } \\
\text { estudo pode ser explicada pela região geográfica } \\
\text { na qual os ensaios foram realizados. }\end{array}$ \\
\hline $\begin{array}{l}\text { Fernández García et al., } \\
2003\end{array}$ & $\begin{array}{l}\text { Efectividad del consejo médico a pacien- } \\
\text { tes alcohólicos y bebedores excesivos } \\
\text { atendidos en consultas de atención } \\
\text { primaria }\end{array}$ & $\begin{array}{l}\text { Verificar a eficácia do aconselhamento médico } \\
\text { antialcoólico dado em consultas em serviços da } \\
\text { APS. }\end{array}$ \\
\hline $\begin{array}{l}\text { Fernández San Martín } \\
\text { et al., } 1997\end{array}$ & $\begin{array}{l}\text { Efectividad del consejo médico breve } \\
\text { para reducir el consumo de alcohol en } \\
\text { bebedores }\end{array}$ & $\begin{array}{l}\text { Avaliar a eficácia do aconselhamento médico } \\
\text { para reduzir o consumo de álcool em homens } \\
\text { que bebem excessivamente. }\end{array}$ \\
\hline Fink et al., 2005 & $\begin{array}{l}\text { An evaluation of an intervention to assist } \\
\text { primary care physicians in screening and } \\
\text { educating older patients who use alcohol }\end{array}$ & $\begin{array}{l}\text { Avaliar o resultado de atendimento médico e de } \\
\text { práticas educativas específicas para pacientes } \\
\text { idosos com relatos de consumo de álcool de } \\
\text { risco na diminuição de riscos e problemas rela- } \\
\text { cionados a eles. }\end{array}$ \\
\hline Giesbrecht, Ferris, 1993 & $\begin{array}{l}\text { Community-based research initiatives in } \\
\text { prevention }\end{array}$ & $\begin{array}{l}\text { Avaliar prós e contras de pesquisas baseadas na } \\
\text { comunidade e as oportunidades de tais projetos } \\
\text { para orientar políticas voltadas a problemas } \\
\text { preveníveis na comunidade. }\end{array}$ \\
\hline
\end{tabular}




\section{Quadro 1. (cont.)}

AUTOR
2006

Guth et al., 2008

Hawkins et al., 2014

Helseth et al., 2018

Israel et al., 1996

Jomar, Abreu, 2012

Kaner, 2010

Kardakis et al., 2018

Knight et al., 2018

Kuerbis et al., 2015

Kypri et al., 2008

\section{TíTULO}

Community-based interventions and alcohol, tobacco and other drugs: foci, outcomes and implications

Brief intervention in alcohol-dependent versus nondependent individuals

Youth problem behaviors 8 years after implementing the communities that care prevention system: a community-randomized trial

Training community-based treatment providers to implement contingency management for opioid addiction: time to and frequency of adoption

Screening for problem drinking and counseling by the primary care physician-nurse team

Intervenções breves para uso problemático de álcool: potencial de aplicação na prática do enfermeiro

NICE work if you can get it: Development of national guidance incorporating screening and brief intervention to prevent hazardous and harmful drinking in England

Implementation of clinical practice guidelines on lifestyle interventions in Swedish primary healthcare - a two-year follow up

Screening and Brief Advice to Reduce Adolescents' Risk of Riding With Substance-Using Drivers

Testing the initial efficacy of a mailed screening and brief feedback intervention to reduce at-risk drinking in middle-aged and older adults: the comorbidity alcohol risk evaluation study

Randomized controlled trial of web-based alcohol screening and brief intervention in primary care

\section{OBJETIVO}

Examinar opções disponíveis para a comunidade local, evidências de eficácia, ligações entre as experiências locais e as iniciativas regionais e implicações para futuras pesquisas e intervenções.

Examinar se um diagnóstico de dependência de álcool, de acordo aos critérios do Manual Diagnóstico e Estatístico de Transtornos Mentais, Quarta Edição, previu a eficácia diferencial de uma intervenção breve (IB).

Testar se o sistema de prevenção de Comunidades que se preocupam (CTC) reduziu o comportamento de risco problemático de adolescentes em toda a comunidade oito anos após a implementação do CTC.

Comparar duas estratégias de implementação do Contingengy Management, uma intervenção psicossocial com foco no comportamento dirigido a consumidores com uso problemático de opioides.

Verificar a eficiência e a aceitabilidade de um método de rastreio para a identificação de bebedores problemáticos e a efetividade de aconseIhamento dado por uma enfermeira em contexto de estilo de vida.

Apresentar a finalidade, os principais elementos e a aplicabilidade das intervenções breves para o uso problemático de álcool, bem como refletir sobre o potencial de aplicação dessas intervenções na prática do enfermeiro.

Descrever o desenvolvimento do primeiro conjunto de orientações nacionais voltadas à prevenção de problemas relacionados ao álcool na Inglaterra.

Avaliar o progresso resultante de dois anos de acompanhamento de intervencões no estilo de vida e usuários de serviços na APS, bem como o uso de guia de intervenções no estilo de vida desses usuários, na prática clínica.

Testar o efeito do aconselhamento por meio de computador, de forma longitudinal, em jovens que reportaram dirigir após fazer uso de álcool ou outras drogas.

Determinar a eficácia inicial de uma triagem enviada pelo correio e uma breve intervenção para reduzir o consumo de álcool entre pessoas com 50 anos ou mais.

Avaliar eficácia da intervenção motivacional baseada na WEB. 
Quadro 1. (cont.)

\begin{tabular}{ll}
\hline AUTOR & TíTULO \\
\hline Lee et al., 2009 & Harm reduction among at-risk elderly \\
& drinkers: a site-specific analysis from \\
& the multi-site Primary Care Research in \\
& Substance Abuse and Mental Health for \\
& Elderly (PRISM-E) study
\end{tabular}

Mclntosh et al., 1997

Midford, 2010

Millstein, Marcell, 2003

Mitchell, et al., 2013

Moore et al., 2011

Moore et al., 2018

Moretti-Pires, Corradi-Webste, 2011

Nair et al., 2015

Nilsen et al., 2006

Ockene et al., 1999

Oliveira, Soares, Batista, 2016 practice

Drug prevention programmes for young people: where have we been and where should we be going?

Screening and counseling for adolescent alcohol use among primary care physicians in the United States

SBIRT for adolescent drug and alcohol use: current status and future directions

Primary care-based intervention to reduce at-risk drinking in older adults: a randomized controlled trial

Prevention of Underage Drinking on California Indian Reservations Using Individual- and Community-Level Approaches

Effectiveness of strategies to implement brief alcohol intervention in primary healthcare. A systematic review

\section{OBJETIVO}

Avaliar a eficácia da intervenção baseada na redução de danos para melhorar o acesso ao tratamento e os resultados clínicos entre os idosos com consumo de risco.

Comparar os efeitos de três breves métodos de redução do consumo de álcool na prática familiar.

Criar uma melhor compreensão dos elementos que maximizam a eficácia dos programas de prevenção, o que pode ser alcançado pelos programas e como podem ser melhorados.

Examinar a prevalência e a qualidade dos serviços de prevenção do uso de álcool oferecidos a adolescentes, nos Estados Unidos.

Avaliar a eficácia da IB em adolescentes que estão em risco de desenvolver transtornos por uso de substâncias.

Examinar se uma intervenção multifacetada entre os pacientes idosos sob cuidados na APS reduziu o consumo de álcool e o consumo de risco, em três e doze meses.

Avaliar intervenções individuais e junto à comunidade para reduzir o consumo de álcool entre Indígenas Americanos Nativos do Alasca, menores de idade, das comunidades rurais das reservas indígenas da Califórnia.

Avaliar processo de implementação de rastreamento e intervenções breves para o uso problemático de álcool (UPA) em serviços da APS do município de Coari, Amazonas.

Verificar se intervenções baseadas em computadores podem ajudar a superar as barreiras de implementação na APS - falta de tempo, treinamento e recursos e possíveis danos ao relacionamento.

Revisar sistematicamente a literatura disponível sobre a implementação de intervenções breves em cuidados à saúde de consumidores de álcool a fim de determinar a eficácia dos esforços de sua implementação.

Determinar se o aconselhamento, nos moldes de intervenção breve, ministrado tanto pelo médico como pela enfermeira, é eficaz, como parte dos cuidados primários de rotina na redução do consumo de álcool por bebedores de alto risco.

Compreender as representações cotidianas de jovens sobre a periferia, com a finalidade de compor os temas para programas midiáticos de educação sobre drogas 
Quadro 1. (cont.)

AUTOR

Oliveira, Soares, Silva, 2016

Oslin et al., 2006

Reiff-Hekking et al., 2005

Ribeiro, 2011

Richmond et al., 1995

Roche et al., 2015

Ronzani, Mota, Souza, 2009

Saitz, 2010

Senft, 1997

Soares, Vargas, 2019

Spoth, Greenberg,

Turrisi, 2008

Stead M et al., 2017

\section{TíTULO}

Pesquisa- ação emancipatória com jovens escolares: relato de experiência

PRISM-E: comparison of integrated care and enhanced specialty referral in managing at-risk alcohol use

Brief physician and nurse practitioner-delivered counseling for high-risk drinking. Results at 12-month follow-up

A medicina geral e familiar e a abordagem do consumo de álcool: detecção e intervenções breves no âmbito dos cuidados de saúde primários

Controlled evaluation of a general practice-based brief intervention for excessive drinking

Addressing inequities in alcohol consumption and related harms

Prevenção do uso de álcool na atenção primária em municípios do estado de Minas Gerais

Alcohol screening and brief intervention in primary care: Absence of evidence for efficacy in people with dependence or very heavy drinking

Brief intervention in a primary care setting for hazardous drinkers

Efetividade da intervenção breve grupal no uso nocivo de álcool na atenção primária à saúde

Preventive interventions addressing underage drinking: state of the evidence and steps toward public health impact
OBJETIVO

Relatar a experiência de utilização da pesquisa-ação emancipatória, de modo a expor suas potencialidades para problematizar a realidade dos jovens participantes.

Determinar a eficácia relativa de dois modelos de cuidados para reduzir o uso de risco de álcool em pacientes com 65 anos ou mais, na APS.

Determinar os efeitos de intervenção breve, de aconselhamento, prestada por médicos ou enfermeiros na APS para a redução do consumo de álcool por bebedores de alto risco.

Avaliar a efetividade das intervenções breves na intervenção a consumidores com uso prejudicial de álcool; confirmar se a formação de médicos em Intervenções Breves para abordar os Problemas Ligados ao Álcool (PLA) contribui para mudar suas atitudes em relação aos consumidores com uso prejudicial de álcool.

Avaliar a efetividade das IB realizadas por clínicos gerais.

Examinar o uso de álcool e problemas relacionados a partir de uma perspectiva de iniquidades e determinantes sociais.

Avaliar a efetividade da implantação de estratégias de triagem associadas às intervenções breves para prevenção do uso abusivo de álcool na APS.

Determinar se existe evidência de eficácia da IB entre indivíduos com dependência de álcool identificados por triagem em ambientes de cuidados primários.

Testar uma intervenção breve para reduzir o consumo de álcool entre bebedores moderados a pesados em um ambiente de cuidados primários.

Verificar a efetividade da intervenção breve grupal realizada por enfermeiros na redução do uso de risco e nocivo de álcool em usuários de um serviço de atenção primária à saúde.

Destacar a necessidade de razões para uma maior atenção às intervenções preventivas baseadas em evidências para jovens sem idade legal para beber álcool; fornecer revisão das intervencões direcionadas ao consumo prejudicial de álcool com evidência de eficácia ou efetividade; discutir as principais descobertas e suas implicacões do ponto de vista da saúde pública.

Explorar contexto, viabilidade e aceitabilidade da IB em projetos de trabalho de jovens. exploring feasibility and acceptability in a

qualitative study 
Quadro 1. (cont.)

\begin{tabular}{|c|c|c|}
\hline AUTOR & TÍTULO & OBJETIVO \\
\hline $\begin{array}{l}\text { Van de Glind IM et al., } \\
2016\end{array}$ & $\begin{array}{l}\text { Exploring the Range of Lifestyle Interven- } \\
\text { tions Used in Dutch Health Care Practi- } \\
\text { ce: A Qualitative Description }\end{array}$ & $\begin{array}{l}\text { Explorar o uso de intervenções voltadas ao estilo } \\
\text { de vida, realizadas ambulatorialmente, com obje- } \\
\text { tivo de cessação do fumo, redução do consumo } \\
\text { de álcool, aumento de atividade física, promoção } \\
\text { de dieta saudável e perda de peso. }\end{array}$ \\
\hline Walton et al., 2014 & $\begin{array}{l}\text { A randomized controlled trial testing } \\
\text { the efficacy of a brief cannabis universal } \\
\text { prevention program among adolescents } \\
\text { in primary care }\end{array}$ & $\begin{array}{l}\text { Examinar a eficácia de uma intervenção breve, } \\
\text { realizada por terapeuta (TBI) ou por computador } \\
(\mathrm{CBI}) \text {, na prevenção do uso de cannabis entre } \\
\text { adolescentes em clínicas de cuidados primários } \\
\text { urbanos }\end{array}$ \\
\hline $\begin{array}{l}\text { Wells, Moonie, Pharr, } \\
2018\end{array}$ & $\begin{array}{l}\text { Association between alcohol screening } \\
\text { and brief intervention during routine } \\
\text { check-ups and alcohol consumption } \\
\text { among adults living in California }\end{array}$ & $\begin{array}{l}\text { Identificar se os níveis de ingestão alcoólica em } \\
\text { adultos estão associados ao aumento de screen- } \\
\text { ing e IB durante exames de rotina. }\end{array}$ \\
\hline
\end{tabular}

Fonte: Elaboração própria.

\section{Discussão}

A análise dos artigos selecionados, guiada pela concepção teórica eleita por este estudo, permitiu delimitar três categorias empíricas, que correspondem ao objeto das práticas em pauta em cada estudo, bem como às concepções que orientam essas práticas.

A primeira categoria refere-se às práticas desenvolvidas para promover mudanças em comportamentos de risco, ou seja, práticas individuais para os que fazem uso considerado de risco, bem como às práticas programas focalizados em populações expostas a maiores riscos. Além de práticas de prevenção e RD para reduzir riscos, também foram previstas medicalização e internação como formas de tratamento.

Na segunda categoria empírica, estão práticas em saúde mais ampliadas do que as da categoria empírica anterior, as que se propõem a considerar, além de características dos indivíduos, também determinantes sociais do uso prejudicial de drogas, buscando dar respostas a vulnerabilidades atinentes aos sujeitos e aos contextos sociais que favorecem o uso prejudicial de drogas.

A terceira categoria empírica se conforma por práticas emancipatórias, que pretendem formular respostas que atinjam necessidades em saúde, ou seja, ações de enfrentamento a desgastes e condições prejudiciais resultantes das formas de inserção dos indivíduos e suas famílias na reprodução social. Ou seja, condições diversas e adversas que acometem as famílias das diversas classes e frações de classes, os grupos sociais, e que estão na base do consumo prejudicial de drogas. De perspectiva marxista, inclui práticas emancipatórias de RD, práticas de educação emancipatória, que instrumentalizam os grupos sociais para compreender as raízes dos problemas de saúde e os fortalecer para o engajamento na luta por políticas públicas na área e por mudanças sociais radicais em prol da igualdade, como as lutas pela legalização e regulamentação das drogas.

A seguir, apresentam-se os estudos selecionados de acordo com as três categorias empíricas.

\section{O comportamento de risco como objeto das práticas}

Os estudos desta categoria foram agrupados em duas subcategorias: estudos que abordam a Intervenção Breve (IB) e estudos que abordam práticas voltadas à prevenção do consumo ou do consumo de risco, envolvendo o usuário de drogas ou outros indivíduos ou lócus de apoio. 


\section{ESTUDOS SOBRE IB}

A IB é uma abordagem de aconselhamento utilizada desde a década de 1970 para controle do uso abusivo do álcool com vistas a melhorar a saúde dos indivíduos. Objetiva incentivar a tomada de decisão na direção de reduzir ou cessar o uso do álcool para evitar agravos à saúde individual. Tal intervenção baseia-se no pressuposto de que o sentimento de responsabilidade deve ser desenvolvido durante o processo para que, conscientemente, haja controle do consumo de álcool considerado disfuncional, de risco. Atualmente, a IB aplicada por médicos e enfermeiros tem também como objetivo a redução do consumo de outras drogas além do álcool14-29.

A maioria dos estudos primários encontrados, ensaios randomizados que avaliam a eficácia da IB para a redução dos níveis de consumo de álcool, não detalhou elementos facilitadores nem barreiras para a implantação das ações. Coerentemente à concepção dominante na formação de trabalhadores da saúde, o enfoque biomédico foi o mais encontrado nesses artigos, enfoque esse que favorece a fragmentação do objeto do trabalho em saúde e o consequente predomínio de preconceitos e estereótipos ${ }^{31}$. Foi também descrita nessa categoria a descontinuidade das ações implan$\operatorname{tadas}^{32}$, devido à rotatividade de trabalhadores da saúde, associada a mudanças políticas de gestão, e também à não adesão de toda a equipe.

Estudos de revisão e ensaios randomizados selecionados descreveram benefícios dessa abordagem na redução do consumo de álcool e dos riscos relacionados a esse consumo, tanto

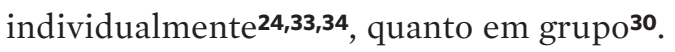
Sugeriram, para fins de maior efetividade, envolvimento de gestores, equipe multidisciplinar, além de integração entre os profissionais da saúde na APS, para a implantação de ações de triagem associadas à IB $^{35}$. A partir da constatação que a IB tem menor eficácia em contextos de condições socioeconômicas desfavoráveis, os autores sugerem o desenvolvimento de mecanismos de fortalecimento da técnica da IB ${ }^{19}$. Destaca-se o guia de práticas criado pelo Institute for Clinical Excellence (Nice), no Reino Unido, que objetiva fomentar práticas multidisciplinares que envolvam acadêmicos, trabalhadores, pacientes e comunidade ${ }^{36}$.

Vários foram os estudos que examinaram aspectos relacionados à eficácia da IB. Entre estes, há o que destaca que é fundamental a qualidade da orientação fornecida pelos profissionais da saúde ${ }^{37}$; outros que afirmam igual eficácia entre a realização da IB por meio de computador (e-IB) e a presencial ${ }^{38}$ ou por telefone ${ }^{39}$. Quanto ao número de sessões de IB, estudo afirmou que intervenções breves de ocasião única ${ }^{40}$ não foram menos eficazes do que sessões adicionais, para o monitoramento de consumo de álcool ao longo do tempo.

Há variação entre resultados de estudos sobre a eficácia da IB entre consumidores de diferentes drogas e com diferentes padrões de consumo. Um deles relata a não eficácia da IB na diminuição de fatores de risco, como abuso de outras drogas e violência ${ }^{41}$. Outro estudo mostrou resultados da eficácia da IB tanto para consumidores com dependência de álcool como para aqueles que fazem uso abusivo da substância ${ }^{42}$. No entanto, revisão sistemática ${ }^{\mathbf{4 3}}$ relatou ausência de evidências que comprovam a eficácia da IB para consumidores com dependência do álcool. Estudos também sinalizaram que a efetividade da IB para a redução do uso de álcool na prática diária ainda não foi bem explorada, com resultados pouco animadores e melhor avaliação da IB em grupos específicos ${ }^{20,21}$.

Estudo desenvolvido na Califórnia identificou baixas taxas, tanto de implementação de screenings quanto de utilização da IB, para uso de álcool, numa relação inversamente proporcional entre a maior ingestão de álcool e a realização de screening e IB. Homens, indivíduos com diabetes e com menor renda que fazem uso de álcool também têm menor probabilidade de acesso à $\mathrm{IB}^{29}$.

Vários são os resultados de estudos que afirmam a necessidade de se adequarem as 
ações para implementação da IB ao público a que será destinada. A população jovem exige estratégias mais acessíveis e atrativas ${ }^{\mathbf{4 4}, \mathbf{4 5}}$; já a população idosa, requer intervenções específicas à faixa etária. Resultados reforçaram a eficácia da IB na modificação do padrão de uso abusivo de álcool ao longo do tempo 46-48.

Quanto ao profissional que realiza a IB na APS, estudo ressaltou a atuação do enfermeiro e a necessidade de ampliação do campo de atuação desse profissional, incentivando o compromisso com a educação em saúde, pois o enfermeiro tem contato constante com usuários, famílias e comunidade ${ }^{49}$.

\section{ESTUDOS QUE ABORDAM PRÁTICAS DE PREVENC̣̃̃O DIRIGIDAS AO INDIVÍDUO CONSUMIDOR DE DROGAS, OU OUTROS INDIVÍDUOS OU LÓCUS DE APOIO}

Nessa categoria empírica, foram descritos estudos que consideram o abuso de álcool e outras drogas como fatores de risco para o aumento do envolvimento dos indivíduos com situações de violência e risco para o desenvolvimento de doenças crônicas ou transmissíveis. Consequentemente, os estudos abordam práticas em saúde na APS com o objetivo de prevenir esse consumo.

No que diz respeito aos resultados das práticas voltadas para a prevenção do consumo de álcool entre jovens, estudo relatou a maior potencialidade daquelas que envolvem a família, a escola, a comunidade e, eventualmente, os locais de trabalho, para identificar situações da vida dos jovens em que o consumo ocorre. Tal possibilidade potencializa a plausibilidade das evidências científicas na orientação da construção de práticas efetivas ${ }^{50}$. A entrevista motivacional e a psicoeducação também mostraram resultados positivos para inibir o consumo de álcool entre jovens indígenas estadunidenses, bem como entre jovens em faixa etária que ainda não respondem legalmente por $\mathrm{si}^{\mathbf{5} 3}$.

Ainda com vistas à prevenção do consumo prejudicial de drogas, foram relatadas práticas para estimular estilo de vida saudável, geralmente associadas à mudança de hábitos, entre estes os relacionados ao uso prejudicial de tabaco e de álcool${ }^{51}$. Os resultados sinalizaram que essas práticas, quando baseadas em manuais aplicados na APS, são mais desenvolvidas por enfermeiros do que por médicos. Práticas que estimulam o estilo de vida saudável, com mudanças de hábitos alimentares e de atividades físicas, com estímulo à perda de peso corporal, são escolhidas quando o foco das intervenções é a abstinência de substâncias $^{52}$. Aposta-se nessa intervenção em consultas regulares em ambulatórios.

As intervenções de RD são analisadas em estudos que têm como objeto os fatores de risco, com a finalidade de reduzir a ingestão de álcool e para incentivar o planejamento para minimizar esse consumo.

Entre idosos, a estratégia de RD apresentou melhores resultados do que os programas que focam na abstinência ${ }^{54}$. Entre jovens, ações pautadas na RD foram desenvolvidas para prevenir o consumo de álcool ou de outras drogas antes de assumirem o volante. As ações foram desenvolvidas nos moldes de programa longitudinal, com aconselhamento auto aplicável por meio de computador - computer-facilitated Screening and Brief Advice (cSBA). $O$ resultado foi a redução das taxas de jovens ao volante após o uso de álcool e de outras drogas, sobretudo entre os que eram responsáveis por sua família ${ }^{55}$.

Programas de prevenção e ações de RD envolvendo a comunidade também foram relatados. Um deles, com enfoque em práticas de RD para potencializar a eficácia de prevenção primária e secundária de consumo de drogas, foi desenvolvido entre escolares, por meio de apoio de pares, em especial o engajamento dos pais, da escola e de outros pontos de apoio da comunidade ${ }^{58-60}$, bem como da promoção de desenvolvimento de habilidades, visando à mudança de comportamento dos jovens ${ }^{56}$. Esses programas tinham como objetivos reduzir taxas de morbimortalidade de adolescentes com consumo abusivo de álcool e prevenir o consumo de drogas entre jovens ${ }^{57}$. 
O delineamento de intervenções baseadas na comunidade mostra-se potente para incorporar práticas baseadas em evidências que abordam preocupações locais de saúde pública ${ }^{61}$. Cita-se a mobilização de atividades de conscientização na comunidade acerca dos problemas relativos ao álcool, bem como restrição da venda dessa droga para os menores de idade 53 .

Para o tratamento de consumidores com uso problemático de opioides, a Contingency Management(CM), uma intervenção psicossocial que usa incentivos motivacionais como gift cards e vouchers, é uma estratégia consolidada. No Canadá, estratégia de implementação da CM, a Science to Service Laboratory (SSL), mostrou bons resultados para mudança de comportamento dos usuários de opioides. No entanto, os resultados não se mostraram melhores quando a intervenção foi desenvolvida por mediadores da intervenção pertencentes a minorias étnico-raciais, sugerindo que questões relacionadas ao contexto sócio cultural podem influenciar na tomada de decisão do mediador da intervenção ${ }^{62}$.

\section{Os determinantes como objeto das práticas}

Nessa categoria empírica, encontra-se uma revisão da literatura que reuniu estudos pautados na concepção multicausal para explicar o consumo de drogas. A revisão apresenta resultados de intervenções sobre determinantes sociais, as que incidem sobre aspectos sociais, como ambiente de trabalho, condições de vida, e iniquidades. Os estudos incluídos identificaram abuso de álcool na infância mais elevado em comunidades desfavorecidas e, em resposta a esse problema, intervenções do tipo visitas domiciliares, educação parental, programas de preparo escolar foram realizadas.

Em relação ao trabalho, identificou-se que indivíduos empregados tendem a beber mais do que aqueles que estão desempregados, uso atribuído à influência por aspectos físicos e psicossociais do trabalho, como a insatisfação dos funcionários, os controles e a cultura do local de trabalho. Intervenções breves, intervenções de pares e psicossociais, treinamento de habilidades e mudança de cultura organizacional podem reduzir o consumo de risco entre os trabalhadores ${ }^{63}$.

A partir de resultados de revisão de artigos, o estudo concluiu que diferentes grupos populacionais têm diferentes padrões de consumo de álcool, influenciados e perpetuados por aspectos estruturais e culturais desses grupos. Por isso, intervenções sob medida, que abordem a desvantagem social dos grupos, por exemplo, ações que incidam na melhoria de condições da habitação, de educação e de trabalho, em determinantes sociais que impactam desfavoravelmente os grupos podem melhorar resultados nas condições de saúde e em padrões de consumo de álcool63.

\section{As necessidades em saúde como objeto das práticas}

Nessa categoria empírica, encontram-se os artigos que relataram resultados de estudos desenvolvidos sob a concepção da teoria da determinação social do processo saúde-doença. Os artigos descreveram pesquisa abordando a temática das drogas, com jovens estudantes que desenvolveram projeto, nos moldes de programa midiático para educação sobre drogas, com metodologia participativa para problematizar o papel do Estado em questões que permeiam a reprodução social - condições de trabalho e consequentes formas de vida - e para identificar a relação intrínseca com o fenômeno produção-distribuição e consumo de drogas na contemporaneidade, no contexto social capitalista de intensa desigualdade social ${ }^{64,65}$.

Os estudos que consideram de maneira formal a dimensão social do problema das drogas distinguem-se entre si, a exemplo do estudo ancorado na concepção multicausal dos determinantes sociais ${ }^{\mathbf{6 1}} \mathrm{e}$ dos que se pautam na concepção da determinação social do processo saúde-doença ${ }^{64,65}$.

Diferenças conceituais entre determinantes social e determinação social vêm sendo debatidas na literatura66-68. 
A concepção dos determinantes sociais propõe a avaliação de vulnerabilidades sociais e, como resposta, ancora ações que incentivem a resiliência dos indivíduos, entre outras mudanças para as quais se pressupõe haver governabilidade por parte dos envolvidos ${ }^{69}$.

De embasamento marxista, a teoria da determinação social interpreta que a base dos mal estares e das necessidades em saúde encontra-se nas condições de reprodução social das classes - grupos sociais - e propõe práticas emancipatórias em resposta a essas necessidades, práticas essas que se propõem ao desafio de apoiar lutas coletivas que fortaleçam grupos sociais submetidos aos problemas de viver em territórios da periferia, frequentemente comandados por milícias ou organizações narcotraficantes ${ }^{69}$.

\section{Limitações do estudo}

O uso de apenas duas bases de dados constitui uma limitação deste estudo. Explorar outras bases e a literatura cinzenta poderia ampliar e diversificar o conjunto de publicações, permitindo outros achados na área.

\section{Implicações para as práticas}

Espera-se que as evidências expostas e discutidas nesta revisão possam subsidiar análises críticas à política de GD, à constituição e à implantação de práticas emancipatórias na área das drogas na APS, fortalecidas e embasadas segundo o referencial emancipatório proposto na categoria empírica que compreende as necessidades em saúde como objeto das práticas em saúde.

\section{Conclusões}

Preponderam nos estudos incluídos nesta revisão práticas voltadas à mudança de comportamentos de risco, o que é congruente com a predominância de intervenções biomédicas nos periódicos indexados nas bases de dados de circulação internacional e nacional e na produção da área da saúde em geral.

A abordagem pela categoria risco não tem compromisso com a exposição de diferenças de classe social. O social, na maioria das vezes, é abordado como um aspecto entre outros de igual ou maior peso, apenas como um fator nebuloso e pouco enfatizado, que aparece como pano de fundo do contexto em questão, que pode influenciar a acentuação da dependência ou ser classificado como um aspecto protetivo ao uso abusivo de drogas. Nessa perspectiva, o usuário é responsável por suas escolhas e pelas mudanças de hábitos ou comportamentos, em direção aos saudáveis.

Vale ressaltar que, embora as práticas sejam orientadas majoritariamente pelo conceito de risco individual, algumas abordagens progressistas mostraram resultados fortalecedores, como é o caso das que envolvem pessoas-chave durante a elaboração e implementação de ações; gestores de saúde, lideranças e comerciantes locais, além de familiares e de outras instituições da comunidade. Nesses casos, a finalidade parece convergir para o fortalecimento do grupo social que está sendo afetado.

Para configurar-se minimamente como ações ou projetos progressistas, é necessário, porém, que sejam planejados e implantados a partir da clareza de sua finalidade, alinhada ao aprimoramento da compreensão do fenômeno das drogas, em vez de alinhar as práticas a objetivos de responsabilização dos indivíduos pelo envolvimento com drogas. Quanto às instituições sociais, devem envolver-se no processo de luta conjunta ao menos pelos direitos sociais de proteção a todos os que moram no território.

A abordagem de RD emancipatória exige posicionamento político progressista e crítico dos envolvidos nos projetos. No âmbito das práticas emancipatórias, os trabalhadores de saúde poderão contribuir com o adensamento dos vínculos entre população e trabalhadores dos serviços, para o aprofundamento da crítica conjunta, com vistas à reivindicação 
de cumprimento, pelo Estado, dos direitos sociais conquistados, ao não desmonte deles, à conquista de novos direitos, mas especialmente à consciência do horizonte da superação das necessidades por meio da luta de classes.

A RD emancipatória fundamenta-se no marco teórico do campo da saúde coletiva, aquele que analisa criticamente o fenômeno das drogas para além da relação drogas-indivíduos e propõe práticas que subsidiem proposições resolutivas ao problema do consumo prejudicial de drogas. Isso significa implantar projetos para serem desenvolvidos por meio de práticas participativas e processuais, ações e práticas que prevejam tempo para aprofundamento analítico e fortalecimento dos grupos sociais que se prejudicam no processo de envolvimento com as drogas, sejam elas legais ou ilegais.

\section{Colaboradoras}

Oliveira LC (0000-0003-3107-2384)* desenvolveu a dissertação de mestrado que deu origem ao manuscrito e trabalhou na sua concepção, pesquisa bibliográfica, síntese, análise dos dados e aprovação do manuscrito. Cordeiro L (0000-0003-2912-1087)* contribuiu para o processo de concepção do manuscrito, atualização da pesquisa bibliográfica, síntese e análise dos dados, revisão e aprovação do manuscrito. Soares CB (0000-0002-8457-3775)* orientou todo o processo de mestrado e colaborou para o processo de concepção, análise dos dados, revisão e aprovação do manuscrito. Campos CMS (0000-0002-1149-9025)* contribuiu para a atualização da pesquisa bibliográfica, síntese e análise dos dados, revisão e aprovação do manuscrito.

\section{Referências}

1. Teixeira MB, Ramôa ML, Engstrom E, et al. Tensões paradigmáticas nas políticas públicas sobre drogas: análise da legislação brasileira no período de 2000 a 2016. Ciênc. Saúde Colet. [internet]. 2017 [acesso em 2018 fev 17]; 22(5):1455-66. Disponível em: http:// dx.doi.org/10.1590/1413-81232017225.32772016.

2. Santos VE, Soares CB, Campos CMS. Redução de danos: análise das concepções que orientam as práticas no Brasil. Physis Rev. Saúde Colet. 2010 [acesso em 2018 fev 17]; 20(3):995-1015. Disponível em: http:// dx.doi.org/10.1590/S0103-73312010000300016.
3. Castro L. A redução de danos à luz do materialismo histórico [dissertação]. Paraná: Universidade Estadual de Maringá, Departamento de Psicologia; 2018. [acesso em 2019 dez 23]. Disponível em: http://www. ppi.uem.br/arquivos-2019/lais-castro-dissertacao.

4. Brasil. Ministério da Saúde, Secretaria Executiva, Coordenação Nacional de DST e Aids. A Política do Ministério da Saúde para atenção integral a usuários de álcool e outras drogas. Brasília, DF: Ministério da Saúde; 2003 [acesso em 2018 fev 17]. Disponível em: http://bvsms.saude.gov.br/bvs/publicacoes/pns_alcool_drogas.pdf.
*Orcid (Open Researcher and Contributor ID). 
5. Brasil. Ministério da Saúde. Portaria GM/MS, $\mathrm{n}^{\circ}$ 2.197, de 04 de Outubro de 2004. Política de Atenção Integral aos usuários de álcool e outras drogas. Brasília, DF: Ministério da Saúde; 2004. [acesso em 2018 fev 17]. Disponível em: http://bvsms.saude.gov.br/bvs/ saudelegis/gm/2004/prt2197_14_10_2004.html.

6. Machado LV, Boarini ML. Políticas sobre drogas no Brasil: a estratégia de redução de danos. Psicol. Ciênc. Prof. 2013 [acesso em 2018 mar 13]; 33(3):580595. Disponível em: https://dx.doi.org/10.1590/S141498932013000300006.

7. Coelho HV, Godoy A, Oliveira E, et al. Políticas públicas de saúde aos usuários de álcool e outras drogas: contribuição da Saúde Coletiva ao debate. Rev. Ciênc. da Saúde da Facul. Estácio de Sá de Goiás. 2012; (2):194-203.

8. Moreira CR, Soares CB, Campos CMS, et al. Redução de danos: tendências em disputa nas políticas de saúde. Rev. Bras. Enferm. 2019 [acesso em 2020 jan 4]; 72(supl3):312-320. Disponível em: http://dx.doi. org/10.1590/0034-7167-2017-0671.

9. Gomes TB, Vecchia MD. Harm reduction strategies regarding the misuse of alcohol and other drugs: a review of the literature. Ciênc. Saúde Colet. 2018 [acesso em 2018 fev 17]; 23(7):2327-2338. Disponível em: http://dx.doi.org/10.1590/1413-81232028237.21152016.

10. Soares CB, Hoga LAK, Peduzzi M, et al. Integrative Review: Concepts And Methods Used In Nursing. Rev. esc. enferm. USP [internet]. 2014 [acesso em 2019 dez 8]; 48(2):335-345. Disponível em: http:// dx.doi.org/10.1590/S0080-6234201400002000020.

11. Soares CB, Campos CMS, Yonekura T. Marxism as a theoretical and methodological framework in collective health: implications for systematic review and synthesis of evidence. Rev. esc. enferm. USP. 2013 [acesso em 2019 dez 8]; 47(6):1403-1409. Disponível em: http://dx.doi.org/10.1590/S0080623420130000600022 .

12. Whittemore R, Knafl K. The integrative review: upda- ted methodology. J Adv Nurs. 2005 [acesso em 2018 fev 17]; 52(5):546-53. Disponível em: https://doi. org/10.1111/j.1365-2648.2005.03621.x 19.

13. Moher D, Liberati A, Tetzlaff J, et al. The PRISMA Group. Preferred reporting items for systematic reviews and meta-analyses: the PRISMA Statement. PLoS Med. 2009 [acesso em $2018 \mathrm{fev}$ 17]; 21;6(7):e1000097. Disponível em: https://doi. org/10.1371/journal.pmed.1000097.

14. Anderson P. Effectiveness of general practice interventions for patients with harmful alcohol consumption. Br J Gen Pract. 1993 [acesso em 2018 jun 26]; 43(374):386-9. Disponível em: https://www.ncbi.nlm. nih.gov/pmc/articles/PMC1372526/.

15. Richmond R, Heather N, Wodak A, et al. Controlled evaluation of a general practice-based brief intervention for excessive drinking. Addiction. 1995 [acesso em 2018 jun 26]; 90(1):119-32. Disponível em: https:// doi.org/10.1046/j.1360-0443.1995.90111915.x.

16. Israel Y, Hollander O, Sanchez-Craig M, et al. Screening for problem drinking and counseling by the primary care physician-nurse team. Alcohol Clin Exp Res. 1996 [acesso em 2018 jun 26]; 20(8):1443-50. Disponível em: http://doi.org/10.1111/j.1530-0277.1996. tb01147.x.

17. Fernández San Martín MI, Bermejo Caja CJ, Alonso Pérez M, et al. Efectividad del consejo médico breve para reducir el consumo de alcohol en bebedores. Aten Primaria. 1997 [acesso em 2018 jun 26]; 19(3):127-32. Disponível em: https://www.elsevier. es/es-revista-atencion-primaria-27-articulo-efectividad-del-consejo-medico-breve-14469.

18. Senft RA, Polen MR, Freeborn DK, et al. Brief intervention in a primary care setting for hazardous drinkers. Am J Prev Med. 1997 [acesso em 2018 jun 26]; 13(6):464-70. Disponível em: https://doi. org/10.1016/S0749-3797(18)30143-0.

19. Córdoba R, Delgado MT, Pico V, et al. Effectiveness of brief intervention on non-dependent alcohol drinkers 
(EBIAL): a Spanish multi-centre study. Fam Pract. 1998 [acesso em 2018 jun 26]; 15(6):562-8. Disponível em: https://doi.org/10.1093/fampra/15.6.562.

20. Fernández García JA, Ruiz Moral R, Pérula de Torres LA, et al. Efectividad del consejo médico a pacientes alcohólicos y bebedores excesivos atendidos en consultas de atención primaria. Aten Primaria. 2003 [acesso em 2018 jun 26]; 31(3):146-53. Disponível em: https://doi.org/10.1016/S0212-6567(03)70674-X.

21. Ballesteros J, Duffy JC, Querejeta I, et al. Efficacy of brief interventions for hazardous drinkers in primary care: systematic review and meta-analyses. Alcohol Clin Exp Res. 2004 [acesso em 2018 jun 26]; 28(4):608-18. Disponível em: http://doi. org/10.1097/01.alc.0000122106.84718.67.

22. Bertholet N, Daeppen J-B, Wietlisbach V, et al. Reduction of alcohol consumption by brief alcohol intervention in primary care: systematic review and meta-analysis. Arch Intern Med. 2005 [acesso em 2018 jun 26]; 165(9):986-95. Disponível em: http:// doi:10.1001/archinte.165.9.986.

23. Beich A, Gannik D, Saelan H, et al. Screening and brief intervention targeting risky drinkers in Danish general practice--a pragmatic controlled trial. Alcohol Alcohol. 2007 [acesso em 2018 jun 26]; 42(6):593603. Disponível em: https://doi.org/10.1093/alcalc/ agm063.

24. Reiff-Hekking S, Ockene JK, Hurley TG, et al. Brief physician and nurse practitioner-delivered counseling for high-risk drinking. Results at 12-month follow-up. J Gen Intern Med. 2005 [acesso em 2018 jun 26]; 20(1):7-13. Disponível em: http://doi.org/10.1111/ j.1525-1497.2005.21240.x.

25. Nilsen P, Aalto M, Bendtsen P, et al. Effectiveness of strategies to implement brief alcohol intervention in primary healthcare. A systematic review. Scand J Prim Health Care. 2006 [acesso em 2018 jun 26]; 24(1):5-15. Disponível em: http://doi. org/10.1080/02813430500475282.
26. Ribeiro C. A medicina geral e familiar e a abordagem do consumo de álcool: detecção e intervenções breves no âmbito dos cuidados de saúde primários. Acta Med Port. 2011 [acesso em 2018 jun 26]; 24(supl2):355-68. Disponível em: https://actamedicaportuguesa.com/ revista/index.php/amp/article/download/1480/1066.

27. Elzerbi C, Donoghue K, Drummond C. A comparison of the efficacy of brief interventions to reduce hazardous and harmful alcohol consumption between European and non-European countries: a systematic review and meta-analysis of randomized controlled trials. Addiction. 2015 [acesso em 2018 jun 26]; 110(7):1082-91. Disponível em: http://doi.org/10.1111/ add.12960.

28. Kuerbis AN, Yuan SE, Borok J, et al. Testing the initial efficacy of a mailed screening and brief feedback intervention to reduce at-risk drinking in middle-aged and older adults: the comorbidity alcohol risk evaluation study. J Am Geriatr Soc. 2015 [acesso em 2018 jun 26]; 63(2):321-6. Disponível em: http://doi. org/10.1111/jgs.13237.

29. Wells GK, Moonie S, Pharr J. Association between alcohol screening and brief intervention during routine check-ups and alcohol consumption among adults living in California. Arch. Psych. Nursing. 2018 [acesso em 2020 fev 2]; (32):872-877. Disponível em: https:// doi.org/10.1016/j.apnu.2018.07.001.

30. Soares J, Vargas D. Efetividade da intervenção breve grupal no uso nocivo de álcool na atenção primária à saúde. Rev Saúde Pública. 2019 [acesso em 2020 fev 2]; 53(2):1-10. Disponível em: https://doi.org/10.11606/ S1518-8787.2019053000498.

31. Moretti-Pires RO, Corradi-Webster CM. Implementação de intervenções breves para uso problemático de álcool na atenção primária, em um contexto amazônico. Rev Lat Am Enfermagem. 2011 [acesso em 2018 jun 26]; 19(esp):813-20. Disponível em: http:// dx.doi.org/10.1590/S0104-11692011000700020.

32. Amaral MB, Ronzani TM, Souza-Formigoni MLO. 
Process evaluation of the implementation of a screening and brief intervention program for alcohol risk in primary health care: An experience in Brazil. Drug Alcohol Rev. 2010 [acesso em 2018 jun 26]; 29(2):162-8. Disponível em: http://doi.org/10.1111/ j.1465-3362.2009.00120.x.

33. McIntosh MC, Leigh G, Baldwin NJ, et al. Reducing alcohol consumption. Comparing three brief methods in family practice. Can Fam Physician. 1997 [acesso em 2018 jun 28]; (43):1959-67. Disponível em: https://www.ncbi.nlm.nih.gov/pmc/articles/ PMC2255191/?page=1.

34. Ockene JK, Adams A, Hurley TG, et al. Brief physician- and nurse practitioner-delivered counseling for high-risk drinkers: does it work? Arch Intern Med. 1999 [acesso em 2018 jun 28]; 159(18):2198-205. Disponível em: http://doi.org/10.1001/archinte.159.18.2198.

35. Ronzani TM, Mota DCB, Souza ICWd. Prevenção do uso de álcool na atenção primária em municípios do estado de Minas Gerais. Rev Saude Publica. 2009 [acesso em 2018 jun 28]; 43(supl1):51-61. Disponível em: http://dx.doi.org/10.1590/S003489102009000800009

36. Kaner E. NICE work if you can get it: Development of national guidance incorporating screening and brief intervention to prevent hazardous and harmful drinking in England. Drug Alcohol Rev. 2010 [acesso em 2018 jun 28]; 29(6):589-95. Disponível em: http:// dx.doi.org/10.1111/j.1465-3362.2010.00236.x.

37. Millstein SG, Marcell AV. Screening and counseling for adolescent alcohol use among primary care physicians in the United States. Pediatrics. 2003 [acesso em 2018 jun 28]; 111(1):114-22.

38. Kypri K, Langley JD, Saunders JB, et al. Randomized controlled trial of web-based alcohol screening and brief intervention in primary care. Arch Intern Med. 2008 [acesso em 2018 jun. 28]; 168(5):530-6. Disponível em: http://dx.doi.org/10.1001/archinternmed.2007.109.

39. Curry SJ, Ludman EJ, Grothaus LC, et al. A randomi- zed trial of a brief primary-care-based intervention for reducing at-risk drinking practices. Health Psychol. 2003 [acesso em 2018 jun 28]; 22(2):156-65. Disponível em: https://doi.org/10.1037/0278-6133.22.2.156.

40. Nair NK, Newton NC, Shakeshaft A, et al. A Systematic Review of Digital and Computer-Based Alcohol Intervention Programs in Primary Care. Curr Drug Abuse Rev. 2015 [acesso em 2018 jun. 28]; 8(2):111-8. Disponível em: https://doi.org/10.2174/1874473708 666150916113538.

41. Walton MA, Resko S, Barry KL, et al. A randomized controlled trial testing the efficacy of a brief cannabis universal prevention program among adolescents in primary care. Addiction. 2014 [acesso em 2018 jun 28]; 109(5):786-97. Disponível em: https://doi.org/10.1111/ add.12469.

42. Guth S, Lindberg SA, Badger GJ, et al. Brief intervention in alcohol-dependent versus nondependent individuals. J Stud Alcohol Drugs. 2008 [acesso em 2018 jun 28]; 69(2):243-50. Disponível em: https:// doi.org/10.15288/jsad.2008.69.243.

43. Saitz R. Alcohol screening and brief intervention in primary care: Absence of evidence for efficacy in people with dependence or very heavy drinking. Drug Alcohol Rev. 2010 [acesso em 2018 jun 28]; 29(6):63140. Disponível em: https://doi.org/10.1111/j.14653362.2010.00217.x.

44. Stead M, Parkes T, Nicoll A, et al. Delivery of alcohol brief interventions in community-based youth work settings: exploring feasibility and acceptability in a qualitative study. BMC Public Health. 2017 [acesso em 2018 jun 28]; 17(1):357-370. Disponível em: https://doi.org/10.1186/s12889-017-4256-1.

45. Mitchell SG, Gryczynski J, Grady KE, et al. SBIRT for adolescent drug and alcohol use: current status and future directions. J Subst Abuse Treat. 2013 [acesso em 2018 jun 28]; 44(5):463-72. Disponível em: https://doi.org/10.1016/j.jsat.2012.11.005.

46. Fink A, Elliott MN, Tsai M, et al. An evaluation of an intervention to assist primary care physicians in 
screening and educating older patients who use alcohol. J Am Geriatr Soc. 2005 [acesso em 2018 jun 28]; 53(11):1937-43. Disponível em: https://doi.org/10.1111/ j.1532-5415.2005.00476.x.

47. Moore AA, Blow FC, Hoffing M, et al. Primary care-based intervention to reduce at-risk drinking in older adults: a randomized controlled trial. Addiction. 2011 [acesso em 2018 jun 28]; 106(1):111-20. Disponível em: https://doi.org/10.1111/j.1360-0443.2010.03229.x.

48. Oslin DW, Grantham S, Coakley E, et al. PRISM-E: comparison of integrated care and enhanced specialty referral in managing at-risk alcohol use. Psychiatr Serv. 2006 [acesso em 2018 jun 28]; 57(7):954-8. Disponível em: https://doi.org/10.1176/ps.2006.57.7.954.

49. Jomar RT, Abreu ÂMM. Intervenções breves para uso problemático de álcool: potencial de aplicação na prática do enfermeiro. Rev enferm UERJ. 2012 [acesso em 2018 jun 28]; 20(3):391-5. Disponível em: https://www.e-publicacoes.uerj.br/index.php/enfermagemuerj/article/view/810.

50. Spoth R, Greenberg M, Turrisi R. Preventive interventions addressing underage drinking: state of the evidence and steps toward public health impact. Pediatrics. 2008 [acesso em 2018 jul 2]; 121(supl4):S311-36. Disponível em: http://doi.org/10.1542/peds.2007$-2243 \mathrm{E}$.

51. Kardakis T, Jerdén L, Nyström ME, et al, Johansson $\mathrm{H}$. Implementation of clinical practice guidelines on lifestyle interventions in Swedish primary healthcare - a two-year follow up. Health Serv Res. 2018 [acesso em 2020 fev 2]; 18:227-240. Disponível em: http:// doi.org/10.1186/s12913-018-3023-z.

52. Van de Glind IM, Heinen MM, Geense WW, et al. Exploring the Range of Lifestyle Interventions Used in Dutch Health Care Practice: A Qualitative Description. Health Promot Pract. 2016 [acesso em 2018 jul 2]; 17(2):235-43. Disponível em: http://doi. org/10.1177/1524839915627264.

53. Moore RS, Gilder DA, Gribe JW, et al. Prevention of underage drinking on Californian Indian Reserva- tions using individual-and-community-level approaches. AmJ Public Health. 2018 [acesso em 2020 fev 2]; 108(8):1035-1041. Disponível em: http://doi. org/10.2105/AJPH.2018.304447.

54. Lee HS, Mericle AA, Ayalon L, et al. Harm reduction among at-risk elderly drinkers: a site-specific analysis from the multi-site Primary Care Research in Substance Abuse and Mental Health for Elderly (PRISM-E) study. Int J Geriatr Psychiatry. 2009 [acesso em 2018 jul 2]; 24(1):54-60. Disponível em: http://doi. org/10.1002/gps.2073.

55. Knight JR, Csemy L, Sherrit L, et al. Screening and Brief Advice to Reduce Adolescents' Risk of Riding With Substance-Using Drivers. J Stud Alcohol Drugs. 018 [acesso em 2020 jan 2]; 79(4):611-616. Disponível em: http://doi.org/10.15288/jsad.2018.79.611.

56. Midford R. Drug prevention programmes for young people: where have we been and where should we be going? Addiction. 2010 [acesso em 2018 jul 2]; 105(10):1688-95. Disponível em: http://doi.org/10.1111/ j.1360-0443.2009.02790.x.

57. Chun TH, Linakis JG. Interventions for adolescent alcohol use. Curr Opin Pediatr. 2012 [acesso em 2018 jul 2]; 24(2):238-42. Disponível em: http://doi. org/10.1097/MOP.0b013e32834faa83.

58. Hawkins JD, Oesterle S, Brown EC, et al. Youth problem behaviors 8 years after implementing the communities that care prevention system: a community-randomized trial. JAMA Pediatr. 2014 [acesso em 2018 jul 2]; 168(2):122-9. Disponível em: http://doi. org/10.1001/jamapediatrics.2013.4009.

59. Giesbrecht N, Ferris J. Community-based research initiatives in prevention. Addiction. 1993 [acesso em 2018 jul 2]; 88(supl):83S-93S. Disponível em: http:// doi.org/10.1111/j.1360-0443.1993.tb02166.x.

60. Giesbrecht N, Haydon E. Community-based interventions and alcohol, tobacco and other drugs: foci, outcomes and implications. Drug Alcohol Rev. 2006 [acesso em 2018 jul 2]; 25(6):633-46. Disponível em: http://doi.org/10.1080/09595230600944594. 
61. Bryant CA, Brown KRM, McDermott RJ, et al. Community-based prevention marketing: organizing a community for health behavior intervention. Health Promot Pract. 2007 [acesso em 2018 jul 2]; 8(2):154-63. Disponível em: http://doi. org/10.1177/1524839906290089.

62. Helseth SA, Janssen T, Scott K, et al. Training community-based treatment providers to implement contingency management for opioid addiction: time to and frequency of adoption. J Subst Abuse Treat. 2018 [acesso em 2020 fev 2]; (95):26-34. Disponível em: http://doi.org/10.1016/j.jsat.2018.09.004.

63. Roche A, Kostadinov V, Fischer J, et al. Addressing inequities in alcohol consumption and related harms. Health Promot Int. 2015 [acesso em 2018 jul 2]; 30(supl2):ii20-35. Disponível em: http://doi. org/10.1093/heapro/dav030.

64. Oliveira E, Soares CB, Batista LL. Representações cotidianas de jovens sobre a periferia. Rev Bras Enferm. 2016 [acesso em 2018 jul 27]; 69(6):1147-53. Disponível em: http://dx.doi.org/10.1590/0034-7167-2016-0387.

65. Oliveira E, Soares CB, Silva JA. Pesquisa-ação emancipatória com jovens escolares: relato de experiência. Rev Gaucha Enferm. 2016 [acesso em 2021 maio 19]; 37(3):e62059-e. Disponível em: https://www.scielo.br/pdf/rgenf/v37n3/0102-6933rgenf-1983-144720160362059.pdf.
66. Rocha PR, David HMSL. Determinação ou Determinantes? Uma discussão com base na Teoria da Produção Social da Saúde? Rev Esc Enf USP. 2015 [acesso em 2018 jul 27]; 49(1):129-135. Disponível em: http:// doi.org/10.1590/S0080-623420150000100017.

67. Spiegel JM, Breilh J, Yassi A. Why language matters: singights and challenges in applying a social determination of health approach in a North-South collaborative research program. Global Health. 2015 [acesso em 2021 maio 19]; (11):9. Disponível em: http://doi. org/10.1186/s12992-015-0091-2.

68. Lopes IO. O discurso da comissão dos Determinantes Sociais da Saúde: avanço político ou retórica social? [dissertação]. São Paulo: Escola de Enfermagem da Universidade de São Paulo; 2017 [acesso em 2018 jul 27]. Disponível em: https://teses.usp.br/teses/disponiveis/7/7141/tde-27042018-112616/publico/dissertacao_final_corrigida.pdf.

69. Oliveira LC, Soares CB, Campos CMS, et al. Práticas emancipatórias na área de drogas: construção de projetos com trabalhadores da Atenção Primária à Saúde. Rev. esc. enferm. USP. 2019 [acesso em 2020 jan 23]; (53):e03528. Disponível em: http://dx.doi. org/10.1590/s1980-220x2018027803528.

\footnotetext{
Recebido em 29/02/2020

Aprovado em 04/08/2020

Conflito de interesses: inexistente

Suporte financeiro: o presente trabalho foi realizado com apoio da Coordenação de Aperfeiçoamento de Pessoal de Nível Superior -

Brasil (Capes) - Código de Financiamento 001
} 